\title{
ROBOTIC PROCESS AUTOMATION: CHALLENGES AND SOLUTIONS FOR THE BANKING SECTOR
}

\author{
Prateek Patri \\ Symbiosis Centre for Information Technology, \\ Pune, Maharashtra, India.
}

\begin{abstract}
In order to cater to the growing customer domain with new service conditions such as $24 x 7$ service, fail-safe service, and mobile service, organizations are incorporating more and more technology-based solutions. Robotic Process Automation (RPA) has emerged as one of the key technology strategies for scaling services with robustness and efficiency. RPA is being increasingly used as a tool to automate, scale-up, manage, analyze, and provide superior customer service. This research paper explains the key challenges banks face in the implementation of RPA and proposes suggestions for banks to avoid these challenges in RPA implementation. The issues faced before and post-implementation of RPA have been discussed. How to deal with security issues on the implementation of RPA has also been discussed. An improved RPA has been proposed to how banks can address new challenges such as debit card fraud and showed how automation benefits the banks in terms of improved error rate, processing time, accuracy, and reliability.
\end{abstract}

Keywords: Robotic Process Automation, Business Process Automation, Banking Industry, Technology in Banking, Work Flow Digitization, RPA Bots, Business Case, RPA Adoption Challenges.

Cite this Article: Prateek Patri, Robotic Process Automation: Challenges and

Solutions for the Banking Sector, International Journal of Management 11(12), 2020, pp. 322-333.

http://iaeme.com/Home/issue/IJM?Volume=11\&Issue=12

\section{INTRODUCTION}

Adaption to digital transformation means the incorporation of new technologies in all aspects of the business which radically affects how one works and provides service to consumers with value. This is also a behavioral change that requires organizations to cope with constant criticism, evaluation, and disappointment. This reorganization of the business process by service-based organizations is called digital transformation. Digital transformation has varying challenges depending on the size of organizations viz. from micro, small and medium organizations to large size organizations. 
The motive behind upgradation of any BPA (Business Process Automation) with inclusion of technology is for improved service efficiency at large scale expansion with maximum business gain. In order to cater for growing customer demand, expansion of business domain and upscaling requirements the service sector need to rediscover efficient techniques. Cost-effectiveness and scale-up being the call of the day for survival for any enterprise. Banking sector is one among the competitive enterprise worldwide rediscovering itself with improved BPA (Business Process Automation) for survival. Increased use of hardware and software robots is the order of the day which gives the benefit of easy scaling, failure free $24 \times 7$ operation with capacity to search large scale digital data and giving efficient business decision support. Therefore, robots and softbots are being increasingly used in strategically key business proves by banking sector to address scaling and efficiency. Although digital transformation is used mainly in a business sense, this impact is also felt from the use of one or more of the current and evolving technologies in other organizations, such as states, government departments, and institutions, to tackle societal problems such as pollution and ageing populations.

Likewise, the banking sector is also a victim of the digital transformation phenomenon. By adopting digitalization, banks can provide upgraded client services. This gives comfort to clients and aids in saving time. Digitalization lessens human blunder and along these lines forges client loyalty. Today, individuals have nonstop access to banks because of internet banking. Overseeing a lot of money has additionally become simpler. Digitalization has likewise profited clients by encouraging cashless exchanges. Clients need not store money any longer and can make exchanges wherever and whenever (Furjan et. al, 2020).

In this process of adaptation, the banking sector has implemented a plethora of new technologies in the business process to make them better, faster, and more secure and among these, one of them is Robotic Process Automation (RPA).

Banking businesses today face growing demands to keep their service as lean as possible whilst offering excellent consumer know-how at the lowest expense. RPA enables large banks to achieve these objectives and stay competitive in a volatile world that is constantly evolving.

RPA can be defined as the technology that currently allows everyone to build computer software or a robot to replicate and incorporate human contact behavior into automated processes for an enterprise. The user interface is used by RPA robots to collect and control data is the same as done manually. RPA bots can translate, trigger responses, and interact with other processes in order to execute various repetitive activities (Jovanović et. al, 2018).

Although the idea of automation of robotics processes was reported in the early 2000s, it was created many years earlier. RPA was built upon three primary innovations and those were: screen scraping, automation of workflow, and artificial intelligence. Screen scraping is the method of data collection from an old user interface in order to view the data in a more current user interface. Workflow automation software provides the benefits of enhancing output, reliability, and precision thereby reducing manual entering of data and increasing order fulfillment speeds. Artificial intelligence involves the capacity of computer systems to execute activities that typically need human interaction.

RPA technology is not part of the organization's IT system of an enterprise. Instead, it remains on top, allowing a company to quickly and effectively introduce the product without disrupting the current processes and facilities. The RPA is a willingness to be conscious of and adjust to shifting contexts, deviations, and new conditions which vary from conventional IT automation. If RPA software is equipped to catch and analyze the behavior of current software applications for different procedures, the program may control data, provide answers, launch new activities, and interact autonomously with other systems. 


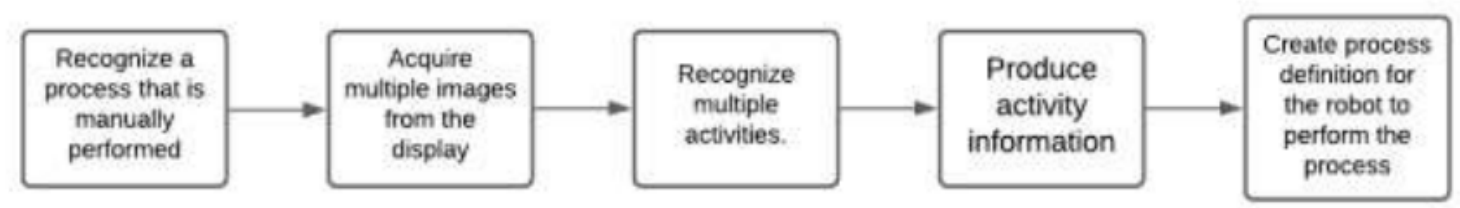

Figure 1. RPA working process

RPA works by running a set of processes as depicted in figure 1. It gives guidelines to the bots on what to do at each stage. When these processes are programmed into the RPA, the product can then consequently run the program and complete the predefined task on various occasions according to the requirements and they communicate with various frameworks through integrations and screen scraping, permitting RPA to perform activities like a desk representative.

The advantages of using RPA technology after conducting the literature review are as follows

- Lowering worker expenses through the automation of frequently performed physical acts.

- The implementation of RPA aims to create successful teams that can carry out complicated activities and assign repetitive jobs to robots.

- No days off and holidays, autonomous robotics will operate $24 \times 7$. RPA algorithms almost totally suppress mistakes and avoid any human influence from happening.

- The typical RPA project repayment duration is 9-12 months, reducing operation delivery costs in effect. The RPA robots require between three to six months to develop and implement.

The processes suitable for automation should follow the criteria such as:

- Low cognitive requirements: Systems with a lot of simple tasks are perfect candidates for RPA implementations, whereas complex processes with a variety of great, complicated tasks are challenging (or impossible) to handle with RPA.

- RPA is applicable to a wide variety of different systems, but usually automates processes or activities within a particular program or information system.

- Effective candidacies for RPA implementations are fairly regular processes and with a high number of activities or tasks carried out.

- Strong chance of human error: Typically, operator-led processes and tasks that present a high risk of human error. The first choice would be to choose RPA implementation methods, as demonstrated by historical data records.

- Limited exception handling: Processes required to be automated using RPA should be processed that have a very low probability of exceptions that are ideal for RPA implementation.

- The purpose of this research paper is:

- To find the challenges that banks face during the adoption of RPA

- To provide a model for banks on what to automate and how to automate so as to realize the maximum benefits of RPA.

\section{LITERATURE REVIEW}

The world of banking is moving towards reducing human error, increased accountability, reducing costs, and being compliant. RPA has become a technology that aims to aid banks in doing so (overcoming these limitations). In this world of compliance, RPA aims to possibly 
disturb the conventional audit model as it can perform rule-based undertakings that are dreary and manual. RPA is relied upon to repurpose the job of the auditing by supplanting cursory errands and stressing on higher-order thinking abilities that will, in the long run, lead to upgraded audit quality (Katke and Kamat, 2019; Moffitt, Rozario, and Vasarhelyi, 2018) as auditors are expected to be unbiased and independent.

RPA is known to the world for its front office processing because it can help industries to eliminate the human involvement in back-offices as they are constantly involved in repetitive work replacing them with bots which reduces spending of the organization. It is also found that a robot can outperform an employee doing the repetitive task by the ratio of 11:1, and in this outperformance, the quality of work is not sullied but has improved too as the task that is completed by the robot is devoid of human error (Anagnoste, 2017). RPA has now also emerged as a threat to low-cost outsourcing as it automates processes very cheaply as a fulltime employee costs around $\$ 80 \mathrm{k}$, an outsourced full-time employee costs around $\$ 30 \mathrm{k}$ but a bot can cost around $\$ 15 \mathrm{k}$ (Petersen and Rohith, 2017).

It is also found that RPA has the power to solve a huge chunk of issues faced by a lot of production companies. The approach suggested is very simple, solving the problem of the manufacturing industry and providing both cost-cutting, producing product performance with completely null errors, and also shifting the workers involved in production work into other positions, where human energy is required to develop the other product-related tasks. For this Optical Character Recognition (OCR) is used which is part of RPA. It mostly helps to recognize the picture and some secret text which further helps to identify where to click and to recognize pictures that are better than blurry photos likewise the likelihood of error is reduced. In summary, the proposed system is $90 \%$ more accurate using RPA than using traditional methods (Lin et at., 2019).

In an RPA framework designed for a CSP (Corporate Service Provider) for its annual compliance processes and ad-hoc client requests, it is reported that the RPA bots will automatically prepare documentation for both annual compliance processes and ad-hoc client request and it will convey the steps necessary and follow-up with the customers. Based on the CSP's assessment system, the overall efficiency gain of the RPA is significantly high (William and William, 2019). It is also reported that RPA can be utilized to incorporate repetitive tasks inside an ERP and automate a huge number of the processes that exist while dealing with a Student Management System. These advancements have been incorporated together inside a website in this manner giving easy access to understand interface, productive ERP, and a chatbot combination. It is conceivable that this integration can be executed in an ever-increasing number of organizations and workplaces. It is demonstrated that it won't just assist the company's productivity by giving information more proficiently, however, it can likewise help in keeping up authority over information, checking information, and its performance (Gajra et al., 2019).

In another article it is published that in the process of existing institutional automation efforts focused on workflow digitization, the application and incorporation of RPA technologies into public administration work processes dramatically improved their productivity, and operation costs and delivered improved services to people. From this finding, it can be inferred that RPA has a tremendous capacity for increasing the performance of institutional work procedures and institutional modernization in general (Houy, Hamberg, and Fettke, 2019).

With the help of current technologies, everyone can access their important tasks including banking online. Bank reconciliation is matching the balances in an accounting record for a cash account to the corresponding information on a bank statement. A bank reconciliation statement (BRS) is a summary of banking and business activity that reconciles an entity's 
bank account with its financial records. The prime aim is to minimize the time and efforts required to reconcile the balance sheets. The system will require minimal manual requirement, by reducing the interactive click by implementing the chatbot. Firstly, they'll receive the information in the form of excel sheet from the bank in which the user details will be stored which will consist of his account number which will be the primary key and using which user details from the database will be fetched. UiPath, Natural language processing tool and AI are implemented in the process. The main aim to integrate UiPath workflows with a chatbot is to make it user-friendly so that the user can query his reconciliation statement, account balance, FAQ and many other such things through it just by authenticating himself. It will also be able to trigger the reconciliation bot and work with all the required excel sheets, thereby reducing the visibility of complexity to the user (Iyer et al., 2019)

RPA is also a modern and evolving recruitment technology and this has enabled the development of various industries. The majority of human resources departments in every company are responsible for several activities along the way. The hiring of the employee till their retirement cycle includes many activities such as repetitiveness, performance, applicant participation, applicant experience, and some of the works still hinges on the Manual method to assist workers in carrying out their jobs. The labor force cycle is expensive and unproductive and often results in a higher rate of mistakes and even the extent of compliance sacrifices. RPA has been found to be responsible for copying repetitive data and other related tasks while HR personnel has an opportunity to concentrate on complex issues of making work-related decisions. In the process of recruitment RPA filters, the profiles of applicants based on the requirement and also communicates with the ones who were not selected explaining the reason via email or text. For efficient incorporation in the process of recruitment, recruiting time should be optimum and cost-efficient. RPA needs to hit the prospect nominee quickly. RPA integration needed to take care of communications, candidate information, sourcing and screening, simple question, and instruction automation. This will lead to the enhancement of quality in the recruitment process. The RPA platform is a very useful tool for streamlining the recruitment process. Recruitment automation allowed the talent acquisition and recruiting professionals to make the right decision more quickly by increasing applicant productivity in the hiring process (Nawaz, 2019)

\section{CHALLENGES}

According to reported literature RPA (Kirchmer, 2017; Pokharkar, 2019; Katke and Kamat, 2019), adoption for the banking industry possess the following challenges:

Selection of the right processes to automate: Organizations frequently face the question of "What to automate?" and "How to automate?" Organizations understand the benefits of automation but the bigger question arises on how to maximize the output by selecting the right process to automate. The integration of RPA with a legacy system is the challenge organizations always face.

Lack of RPA professional talent: The current talent pooling in the banking industry doesn't possess the required talent for automation as the majority of employees are from a non-technical background.

The reputational damage of the organization, outside and within itself: It is the social effect of automation that makes it an urgent reputational issue for organizations considering the creation and utilization of RPA, this makes it even harder for the banking sector as they operate on the trust of society and within the organization, employees may have the fear of losing their jobs due to changes in business processes.

Security: The society has a lot of trust that banks will keep their money secure with them. This hardgained trust will break easily if the bank has a security breach. As the number of cybercrime incidents increases day by day, banks and financial services are easy targets by such cyber criminals as they deal with money. RPA does give these threat agents a new attack surface and the vulnerability of these bots is very high as they need to have privileged access to all ERP modules of the organization. 


\section{4 .SOLUTIONS AND SUGGESTIONS}

Based on the literature it is found that the implementation of RPA possesses few challenges but taking history into account the banking sector has faced all its challenges head-on. With the help of literature available and interviews conducted, the framework is proposed so that banks can avoid these challenges and maximize their RPA benefits.

\subsection{BEFORE the Implementation of RPA}

- It is found that organizations with a culture of innovation have a smoother implementation of RPA than the ones that do not and banks need to make their employees realize that RPA is not a threat. The impact of automation demonstrated that people with jobs that include manual or physical errands had increasingly adverse reactions concerning the effect of automation on their professions, though those employed as a manager and in data sciences revealed progressively constructive perspective. Banks can also recommend internal structural changes in RPA adoption since for their most successful implementation banks have organized themselves differently for innovative ideas and implementations (Choi and Baker, 2017; Sciulli, 1998). Though there is great hype created for the values and business that RPA automation is going to bring; with expanding use cases, there is as yet slack in the standard selection of RPA innovation in the financial business. Fortunately, there are developments and evolutions in the field of technology that has the potential to address these challenges. Artificial Intelligence (AI) and Robotic Process Automation (RPA) Revolution can be the innovation that can be driven into the Banking industry to address the aforementioned challenges. By 2025, robotics will carry out $45 \%$ of development activities. By 2020, the demand for robotics and AI solutions has been valued by Merrill Lynch at 153billion dollars. Robo consultants are a big transformative tool for mainstream wealth management with a capacity of 255 billion USD in Assets Under Management (AUM) by 2018 (Kumar, and Balaramachandran, 2018).

- Successful RPA implementation at an organization named Xchanging was because they had an RPA project sponsor i.e. who enables the success of a project the person or group that gives support and resources for a project or program and an RPA project champion i.e. who takes the responsibility of the entire project team that guides to the success of a project giving a respectable portion of their time to the implementation of RPA at their organization. The chances of successful implementation shoot up if banks do appoint deserving candidates in such roles to maximize their RPA investment (Willcocks, Lacity, and Craig, 2015).

- In daily business practices, the optimal implementation of RPA has become a need. In the near future, businesses will not survive in a competition of industry if they do not incorporate this innovation into their operations. RPA has evolved into modern company vocabulary and is among the best innovations/invention of the 21 st century for a better tomorrow. The methodology of day to day business of organizations and how employees' complete tasks assigned to them will be improved (Madakam, Holmukhe, and Jaiswal, 2019).

- RPA should be used for long repetitive tasks to streamline business processes. RPA is not that robust or reliable in automating processes if it involves a human element or if it is fed with data of substandard quality and scanty business rules. By following these simple rules organizations stand to maximize their automation benefits. Xchanging during their implementation made it a point to first stabilize the processes before automation by using basic six sigma principles as automating unstable processes 
might be counteractive to the achieving RPA benefits. A business approach and methodology known by the name of six sigma that leads to revenue growth breakthroughs through quantifiable gains in product quality, customer satisfaction, and productivity. In the 1980s Motorola developed the concept of implementing Six Sigma processes and aimed at reducing the number of defects to a minimum of 3.4 parts per million opportunities. It is seen the concept of six sigma was successfully implemented in Bank of America as part of their process improvement initiative (Willcocks, Lacity, and Craig, 2015; Houy, Hamberg, and Fettke, 2019).

- In the initial phase, UTILITY an organization in the energy sector had a small team focused on RPA in its organization but as time progressed through internal training the RPA team grew and made the transition even smoother (Willcocks, Lacity, and Craig, 2015). Banks could also use a similar approach and use upskilling programs to evolve the RPA teams. Upskilling programs have economic, social, and individual benefits for the organization as well to the employees (Jarrad and Hudson, 2017). This can be done by making training programs readily accessible and affordable for all the employees, and employees get to choose their training mode of training i.e. online, self, or classroom training.

\subsection{POST Implementation}

During the initial implementation of RPA at telephonica O2, the organization faced issues as their infrastructure was not in place, and took weeks in to just get RPA up to the desired speed. Banks should already have their infrastructure in place and once RPA moves to a strategic level infrastructure should be optimized as well (Willcocks, Lacity, and Craig, 2015).

RPA bots typically are given specific tasks but when these bots are not working, they can be given completely different tasks, this does not cost banks extra and they stand to maximize their RPA investment. As RPA bots can work $24 \times 7$ if they are assigned tasks that take 8 hours a day, the same bot can be used for a different task for the rest of the 16 hours. The concept of a multiskilled workforce is existent for a long time using the same concept, we can enhance the productivity of RPA and not the drawbacks of a multiskilled workforce i.e. training costs, lack of focus by employees on the task at hand are eliminated due to the nature of bots (Willcocks, Lacity, and Craig, 2015).

\subsection{Security Issues}

The RPA framework must be restricted to the least privileged yet, also the data created with the accreditations of the RPA framework ought not to be trusted totally and must be confirmed by RPA professionals. The organization must assess the current condition of possible risks and protections also by progressing improvement through patches for the RPA systems all the time. Organizations should guarantee that security is in scope during development and vulnerabilities do not need to be fixed by extra programming or need to be fixed after implementation of the RPA bots (Buyens, Scandariato, and Joosen 2013). In an interview conducted, recently RPA has helped the financial sector a lot as blue prism has shown clear signs of success in achieving higher-level security with the help of RPA. DTCC (Depository Trust and Clearing Corporation) provides clearing and settlement services to American stock markets. An expanding number of cyber threats, including phishing emails and pernicious URLs, drove DTCC to look for an innovative answer to address the threats and give the organization a safer and increasingly secure work condition. A cross-functional team implemented Blue Prism's connected-RPA along with APIs and Machine Learning to assess the severity of these threats. Digital Workers have increased processing volumes-including during peak times - and decreased the risk to DTCC by focusing on near real-time threat analysis. The Digital Workforce enabled the organization to focus on threat prevention which 
improved client experience and up-levelled employee satisfaction creating a more secure work environment. Such a level of success can be achieved once RPA is implemented in an organization's business process with the benefit of a higher level of security.

It is found through interviews that bots have a set of credentials that is updated on a specific time e.g. monthly, but RPA bots tend to have special permissions assigned to them, if attackers get access to such credentials the impact faced by banks will be severe. So, in figure 2 below we have suggested a system where we eliminate this issue. RPA bots will get temporary access to systems to perform tasks on by a credential management system, this system will have to maintain logs of all RPA access requests, session durations, session terminations for further audits. This management system will terminate the session when the duration is allotted to the bot and the bot is logged out of the system. For example, if an RPA bot wants access to a particular system according to its rules it will first request for permission to the credential management system. If the request is approved it is allowed to perform its tasks on the system for 8 hours, once the completion of 8 hours the session is terminated and the bot loses access to the system. For each request the bot is given different credentials and it is made sure that it is not the previous one.
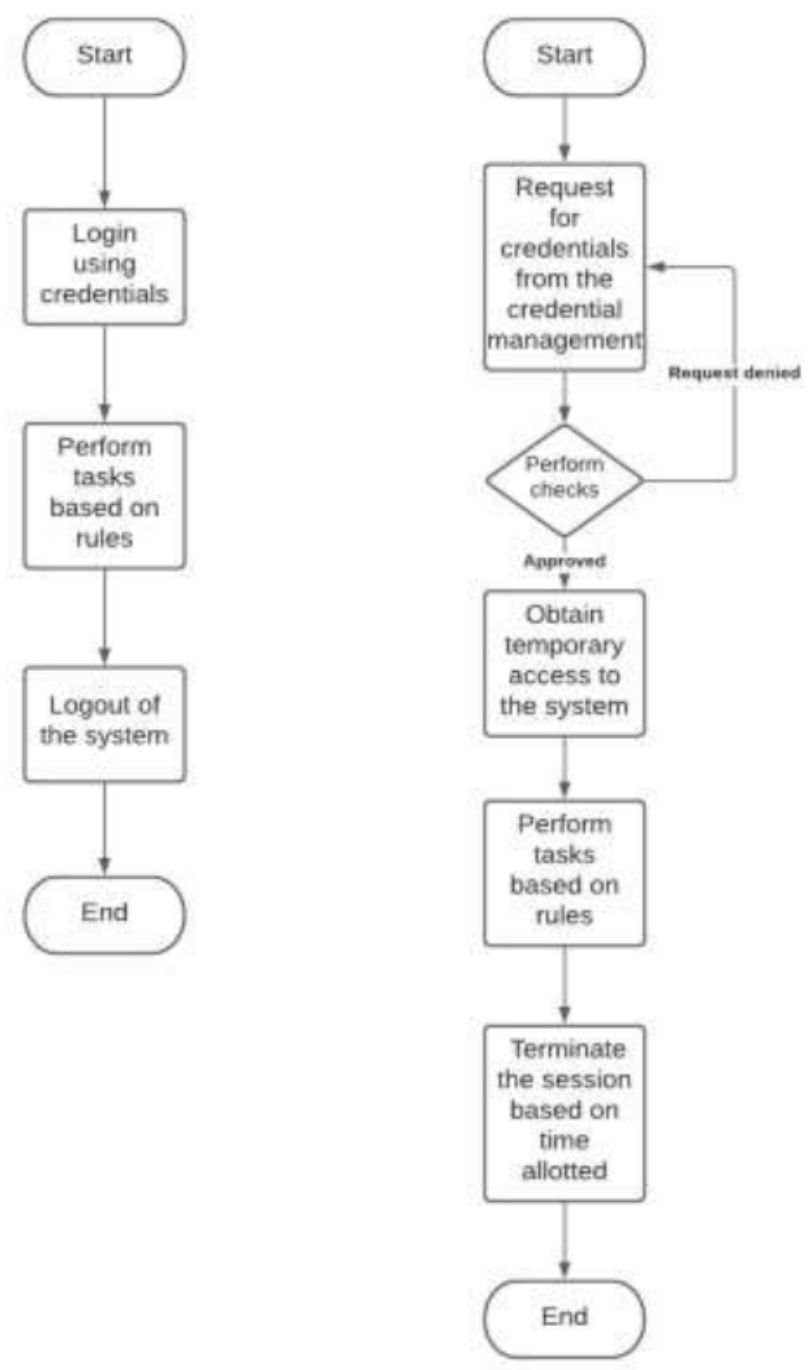

Figure 2. RPA Bot process pre (left) and post (right) access management system 


\section{BUSINESS CASE}

Debit cards are vehicles that facilitate cashless transfers. A host of transactions may be carried out with a debit card. Debit cards will save you, be it online or in grocery shopping or paying utility bills. One can even withdraw cash via a debit card from his account. This process results in a farewell to long lines at the bank or holds to withdrawal slips.

Debit cards are, however, still vulnerable to theft. The planet has been storming with digital payments and simultaneously, debit card theft is common all over the world. In the financial world a hot card is a debit or credit card that cannot be used unless it is stated to have been misplaced or robbed and when a criminal or someone other than the owner wants to use a hot card, the transaction will be denied. We have designed the business process of how banks can process debit card fraud and showed how automation benefits the banks in terms of error rate (error rate $=0$ in the case of automation), processing time, accuracy, and reliability of the process. For effective implementation of automation, banks are supposed to have in place is the bank's work culture and an innovative workforce for automation. To set the automation of this process it will take 2-3 weeks hence a team headed by a project manager is required and, it should be in compliance with all banking and finance regulations. The RPA bot here will first acquire credentials and then navigate and login into the bank's transaction system using its credentials, will select all the hot card data required and export it to an excel sheet and get rid of any redundancies possible, login into the bank's transaction management system, import the excel sheet and process each instance using the rules it is programmed to do. Referring the figure 3 drawn below we realize that the payoff of injecting robots will result in zero error whereas in the manual process the chances of error are likely to be significant, the chances of fraud committed to handling this automation process have been reduced highly, increased service and reliability provided to customers. After conducting interviews with some bank employees, we have understood the decisions taken and time spent per process and an employee process around 100 fraud cases a day or less depending on the efficiency of the employee whereas an RPA bot can process around close to 1000 fraud transactions a day. These fraud processing bots can also do other banking related tasks when not in use. 


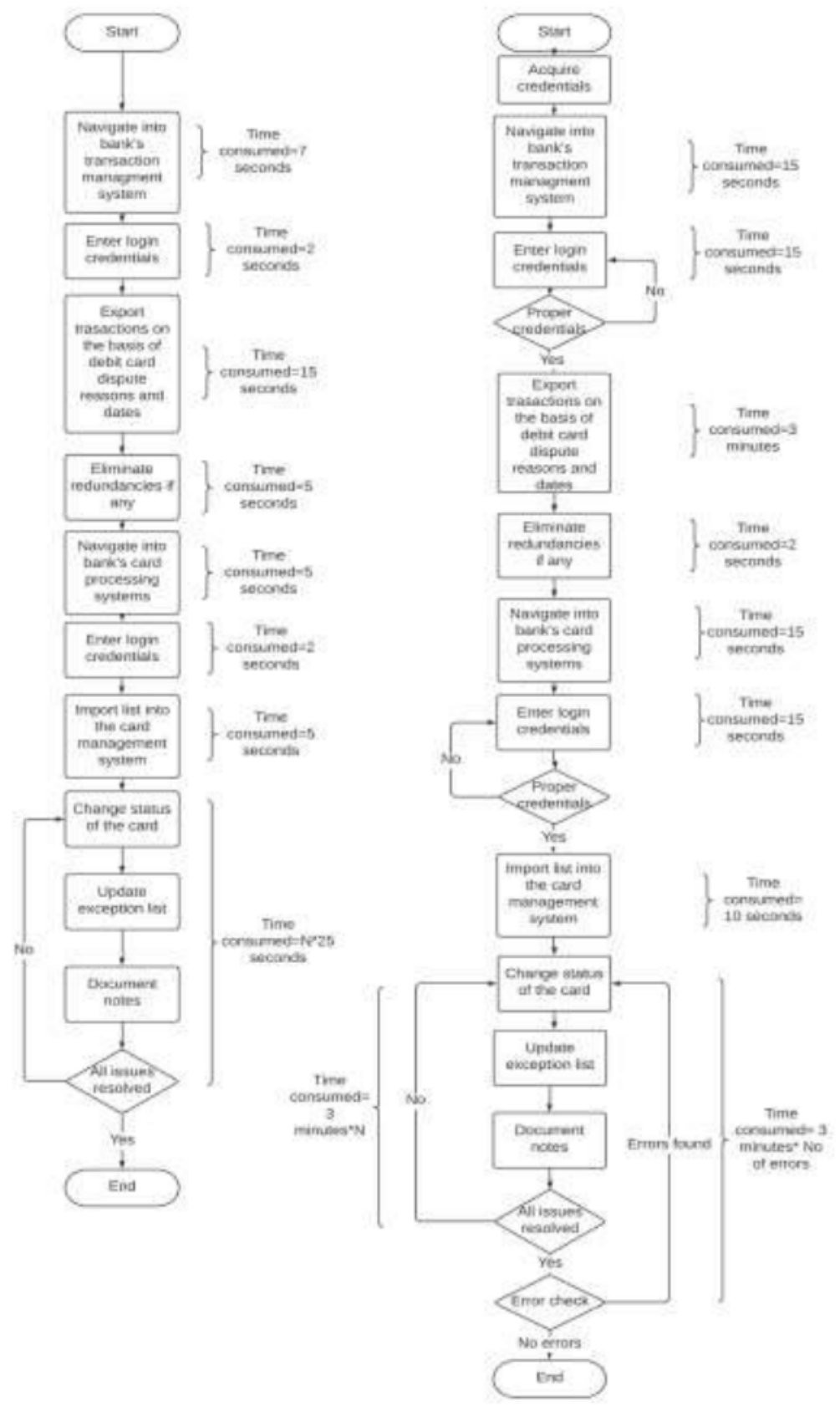

Figure 3. Automated process (Left) and Manual process (Right)

\section{CONCLUSION}

Automation in the banking sector is an important step towards digital transformation. This transformation will not only aid in saving time but also provide flawless service. RPA is a type of automation in which a robot or through computer programming is possible to carry out the activities that a man/woman can do. RPA works by running a set of process and it gives a lot of benefits to a bank along with improvement in quality, scalability, and resiliency in cost-effective means. For the implementation of RPA training of employees is needed. The security of the money deposited by the client should be kept in mind while adopting RPA. 


\section{REFERENCES}

[1] Anagnoste, S. (2017). Robotic Automation Process - The next major revolution in terms of back-office operations improvement. Proceedings of the International Conference on Business Excellence, 11(1), 676-686 https://doi.org/https://doi.org/10.1515/picbe-2017-0072

[2] Choi, Y., and Baker, B. (2017). The impact of automation on business and employment in South Korea. IUP Journal of Supply Chain Management, 14(4), 23-37.

[3] Dodel, M., and Mesch, G. S. (2020). Perceptions about the impact of automation in the workplace. Information, Communication and Society, 23(5), 665-680.

[4] Furjan, M., Tomičić-Pupek, K., and Pihir, I. (2020). Understanding Digital Transformation Initiatives: Case Studies Analysis, Business Systems Research Journal, 11(1), 125-141. doi: https://doi.org/10.2478/bsrj-2020-0009.

[5] Gajra, Vrushil and Lakdawala, Khwajaavais and Bhanushali, Rahul and Patil, Sunita, Automating Student Management System Using ChatBot and RPA Technology (April 8, 2020). Proceedings of the 3rd International Conference on Advances in Science and Technology (ICAST) 2020.

[6] Houy, C., Hamberg, M. and Fettke, P., (2019). Robotic Process Automation in Public Administrations. In: Räckers, M., Halsbenning, S., Rätz, D., Richter, D. and Schweighofer, E. (Hrsg.), Digitalisierung von Staat und Verwaltung. Bonn: Gesellschaft für Informatik e.V.. (S. 62-74).

[7] Iyer, Nilesh and Gori, Harsh and Kumawat, Diksha and Rote, Uday, Bank Reconciliation Bot (April 12, 2019). 2nd International Conference on Advances in Science \& Technology (ICAST) 2019 on 8th, 9th April 2019 by K J Somaiya Institute of Engineering \& Information Technology, Mumbai. http://dx.doi.org/10.2139/ssrn.3370760

[8] Jarrad, S., and Hudson, W. (2017). Staff engagement and upskilling enhances job, care quality Australian Journal of Dementia Care, 4(2).

[9] Jones Jr, M. H. (2004). Six Sigma... at a Bank? Lean and Six Sigma Review, 3(2), 13.

[10] Jovanović, S. Z., Đurić, J. S., and Šibalija, T. V. (2018). Robotic process automation: overview and opportunities. Int. J. Adv. Qual, 46.

[11] Katke, K., G, V., and Kamat, A. (2019). Challenges of robotic process automation adoption in banking and financial services.

[12] Kirchmer, M. (2017). Robotic Process Automation - Pragmatic Solution or Dangerous Illusion? BTOES Insights (Business Transformation and Operational Excellence Summit Insights).

[13] Kumar, K. N., and Balaramachandran, P. R. (2018). ROBOTIC PROCESS AUTOMATIONA STUDY OF THE IMPACT ON CUSTOMER EXPERIENCE IN RETAIL BANKING INDUSTRY. Journal of Internet Banking and Commerce, 23(3), 1-27.

[14] Lacity, M., Willcocks, L., \& Craig, A. (2015). Robotic process automation: mature capabilities in the energy sector. LSE Research Online Documents on Economics

[15] Lacity, M., Willcocks, L., \& Craig, A. (2015). Paper 15/02 Robotic Process Automation at Telefónica O2. The Outsourcing Unit Working Research Paper Series 
[16] Least privilege analysis in software architectures. Software and Systems Modeling, 12(2), 331-348.

[17] Madakam, S., Holmukhe, R. M., and Jaiswal, D. K. (2019). The future digital workforce: Robotic process automation (RPA). JISTEM-Journal of Information Systems and Technology Management, 16.

[18] Moffitt, K. C., Rozario, A. M., and Vasarhelyi, M. A. (2018). Robotic Process Automation for Auditing. Journal of Emerging Technologies in Accounting, 15(1), 1-10. https://doi.org/10.2308/jeta-10589

[19] Nawaz, N. (2019). Robotic process automation for recruitment process. 10, 608-611. https://doi.org/10.34218/IJARET.10.2.2019.057

[20] Petersen, B. L., and Rohith, G. P. (2017). How Robotic Process Automation and Artificial Intelligence Will Change Outsourcing. Brussels, Mayer Brown.

[21] Pokharkar, A. P. (2019). Robotic Process Automation: Concept, Benefits, Challenges in Banking Industry. IIBM'S Journal of Management, 17-25.

[22] S. C. Lin, L. H. Shih, D. Yang, J. Li,n and J. F. Kung, "Apply RPA (Robotic Process Automation) in Semiconductor Smart Manufacturing," 2018 e-Manufacturing and Design Collaboration Symposium (eMDC), Hsinchu, 2018, pp. 1-3.

[23] Sciulli, L. M. (1998, Spring). How organizational structure influences success in various types of innovation. Journal of Retail Banking Services, 20(1), 13+.

[24] W. William and L. William, "Improving Corporate Secretary Productivity using Robotic Process Automation," 2019 International Conference on Technologies and Applications of Artificial Intelligence (TAAI), Kaohsiung, Taiwan, 2019, pp. 1-5, doi: 10.1109/TAAI48200.2019.8959872.

[25] Willcocks, L. P., Lacity, M., and Craig, A. (2015). Robotic process automation at Xchanging. 